\title{
四个天然异戊烯基黄酮的全合成研究
}

\author{
左武标杨金会* 李红俊郭冬冬 \\ 落俊山黄文倩 \\ (宁夏大学天然气转化国家重点实验室培育基地 银川 750021)
}

\begin{abstract}
摘要 利用温和的方法进行了异戊烯基黄酮( \pm )-abyssinone-VI-4- $O$-methyl ether, ( \pm )-abyssinone-IV-4'-O-methyl ether, ( \pm )-abyssinone-V-4'-O-methyl ether 和 $( \pm$-sigmoidin $\mathrm{E}$ 的全合成研究，同时通过对羟基苯甲醛的异戊烯基化以及在石油 醚中低温结晶的方法合成了关键中间体 4-羟基-3,5-二异成烯基苯甲醛. 所有新化合物的结构都经过 IR, ${ }^{1} \mathrm{H}$ NMR, MS, HRMS 确认.
\end{abstract}

关键词 异戊烯基黄酮; (土)-abyssinone-VI-4-O-methyl ether; ( \pm -abyssinone-IV-4'-O-methyl ether; $( \pm$ )-abyssinoneV-4'-O-methyl ether; $( \pm)$-sigmoidin E; 全合成

\section{Study on Total Synthesis of Four Natural Prenylated Flavonoids}

\author{
Zuo, Wubiao Yang, Jinhui* \\ Li, Hongjun \\ Guo, Dongdong \\ Luo, Junshan \\ Huang, Wenqian
}

(State Key Laboratory Cultivation Base of Natural Gas Conversion, Ningxia University, Yinchuan 750021)

\begin{abstract}
A facile approach for the total synthesis of prenylated flavonoids, $( \pm$ )-abyssinone-VI-4- $O$-methyl ether (1), ( \pm )-abyssinone-IV-4'-O-methyl ether (2), ( \pm )-abyssinone-V-4'-O-methyl ether (3) and ( \pm )-sigmoidin E (4), has been described. The key intermediate 4-hydroxy-3,5-di-(3-methylbut-2-enyl)benzaldehyde (6) was synthesized that features regioselective prenylation of 4-hydroxybenzaldehyde and crystallizing with petroleum ether from the reaction mixture by freeze-out effect. All structures of new compounds were confirmed by IR, ${ }^{1} \mathrm{H}$ NMR, MS and HRMS techniques.
\end{abstract}

Keywords prenylated flavonoind; ( \pm )-abyssinone-VI-4- $O$-methyl ether; ( \pm )-abyssinone-IV-4'-O-methyl ether; ( \pm )abyssinone-V-4'-O-methyl ether; ( \pm )-sigmoidin $E$; total synthesis

天然黄酮化合物广泛存在于植物界, 数量众多, 结 构复杂, 具有许多重要的生理和药理活性, 比如: 抗

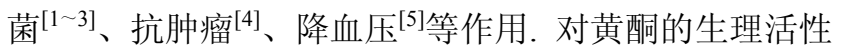
研究, 延等 ${ }^{[6]}$ 做了详细介绍. 他们中的一些已经被当 作药物来使用 ${ }^{[7]}$.

化合物 1 4(图 1)是从刺桐 (Erythrina) 类植物中分 离出来的 ${ }^{[8 \sim 11]}$. 刺桐家族以乔木、灌木为主, 广泛分布 在热带、亚热带地区，我国的华南及四川地区也有广泛 栽培. 虽然黄酩化合物有许多的生理药理活性, 但是自 然界存在的黄酮远远满足不了人们的需要, 因此通过人 工合成黄酮化合物变得尤为重要. 目前化合物 $1,3,4$ 的
合成还未见报道，同时本文对已合成的化合物 2 进行了 合成方法改进，通过更加简单温和的方法合成了化合物 2, 它们的全合成研究在异戊烯基黄酮类合成研究方面 具有重要的意义. 本文通过简单的异戊烯基化, 羟基保 护，羟醛缩合，环合，脱保护等 5 步反应合成了化合物 $1 \sim 4$.

\section{1 结果与讨论}

我们研究小组一直关注黄酮化合物的研究, 并且完 成了一些天然黄酮化合物的合成 ${ }^{[12 ~ 14]}$, 基于这些研究, 我们完成了天然异戊烯基黄酮化合物 $\mathbf{1} \sim \mathbf{4}$ 的全合成

\footnotetext{
*E-mail: yang_jh@nxu.edu.cn

Received October 13, 2012; revised October 31, 2012; published online November 2, 2012.

Project supported by the National Natural Science Foundation of China (Nos. 21162021, 20962016), the Ningxia Natural Science Foundation (No. NZ1006), the Program for New Century Excellent Talents in University (No. NCET-09-0860) and the National Basic Research Program 973 of China (No. 2010CB534916).

国家自然科学基金(Nos. 21162021, 20962016)、宁夏自然科学基金(No. NZ1006)、教育部 “新世纪优秀人才支持计划资助” (No. NCET-09-0860)、国 家重点基础研究发展计划(No. 2010CB534916)资助项目.
} 
<smiles>CCCCCCCCCCCCC(=O)c1ccc(O)cc1O</smiles>

( \pm )-abyssinone-VI-4-O-methyl ether

1<smiles>CCCCCCCC</smiles>

( \pm )-abyssinone-V-4'-O-methyl ether<smiles>CCCCCCCC</smiles>

( \pm )-abyssinone-IV-4'-O-methyl ether 2<smiles>CCCc1cc(C2CC(=O)c3c(O)cc(O)cc3O2)cc2c1OC(C)(C)C=C2</smiles>

$( \pm)$-sigmoidin $\mathrm{E}$

3<smiles>CC(C)=CCC#P</smiles>

图 1 化合物 1 4 的结构

Figure 1 Structures of compounds $\mathbf{1} \sim \mathbf{4}$

(Scheme 1). 对化合物 $\mathbf{5}$ 进行异戊烯基化, 通过两个步骤 得到化合物 6. 将化合物 $\mathbf{6}$ 和硫酸二甲酯溶于丙酮, 加热 回流得到化合物 7. 在碱性条件下，化合物 7 和 13 通过 羟醛缩合反应生成查尔酮 $\mathbf{8}$, 然后进行水解得到天然化 合物 $\mathbf{1}$, 将化合物 $\mathbf{1}$ 溶于无水乙醇, 在无水醋酸钠催化下 环合得到天然化合物 $\mathbf{2}$. 用相同方法, 以化合物 $\mathbf{7}$ 和化合 物 15 为原料, 通过着弪醛缩合、环合、水解等反应合成了 天然化合物 3. 化合物 6 在二氯二氧基苯醌(DDQ)作用 下, 在苯中回流环合生成化合物 $\mathbf{1 0}$, 化合物 $\mathbf{1 0}$ 和化合物 15 通过羟醛缩合, 环合, 水解等反应合成了天然化合物 4. 化合物 1,3,4 的合成研究尚未见报道, 而化合物 2 的 合成工作已有报道 ${ }^{[15]}$, Farmer 等 ${ }^{[15]}$ 通过 Claisen 重排等 总共 12 步反应合成化合物 2, 而本小组仅通过基团保 护、羟醛缩合、环合、脱保护等 5 步反应合成了化合物 2, 反应步骤更加简单, 反应条件更加温和且好控制.

此合成的关键是中间体 4-着基-3,5-二异戊烯基苯甲 醛(6)的合成(Scheme 2). 虽然 Kazuaki 等 ${ }^{[16]}$ 报道了对羟 基苯甲醛的异戊烯基化反应，并且成功合成了此中间 体, 但我们小组的研究发现, 在对羟基苯甲醛的异戊烯 基化的过程中, 虽然可以得到 4-羟基-3,5-二异成烯基苯 甲醛, 但是同时存在的多种副产物以及与之极性相同的 化合物 4- $O$-异戊烯基苯甲醛为分离带来了困难, 简单用 一般的硅胶柱层析方法分离效果并不理想, 因此我们小 组对分离方法进行了改进, 通过两个步骤顺利分离出了 4-羟基-3,5-二异戊烯基苯甲醛：(1)以 $V$ (乙酸乙酯)： $V$ (石 油醚 $)=1: 10$ 将对羟基苯甲醛异戊烯基化后的混合物 进行分离, 去除未反应的原料; (2)将不含原料的混合物 在低于 $0{ }^{\circ} \mathrm{C}$ 环境中冷冻 $24 \mathrm{~h}$, 析出白色固体即我们所需
要的关键中间体 4-差基-3,5-二异戊烯基苯甲醛.

\section{2 实验部分}

\section{1 仪器与试剂}

熔点用 X-5 型熔点测定仪(温度计未校正); IR 用 FTIR-8430S 型红外光谱仪测定 ( $\mathrm{KBr}$ 压片法); NMR 用 Bruker AM-400 MHz 型核磁共振仪测定 $\left(\mathrm{CDCl}_{3}\right.$ 作溶剂, TMS 为内标); $\mathrm{MS}$ 用 5975C 型质谱仪测定; HRMS 用 CAB073；200 300 目及 $\mathrm{GF}_{254}$ 硅胶(青岛海洋化工厂生 产); 试剂均为分析纯.

\section{2 实验方法}

\subsubsection{4-羟基-3,5-二异戊烯基苯甲醛(6)的合成}

在 $0{ }^{\circ} \mathrm{C}$ 下, 向对羟基苯甲醛 $305.0 \mathrm{mg}(2.5 \mathrm{mmol})$ 中 加入 $1 \mathrm{~mol} \cdot \mathrm{L}^{-1}$ 氢氧化钾水溶液 $5.0 \mathrm{~mL}$, 摚拌 $10 \mathrm{~min}$ 后 缓慢滴加异戊烯基溴 $0.6 \mathrm{~mL}(5.0 \mathrm{mmol})$, 继续摚拌 $24 \mathrm{~h}$, 用 $3.0 \mathrm{~mol} \cdot \mathrm{L}^{-1}$ 的盐酸调节 $\mathrm{pH}<2$, 用 $30.0 \mathrm{~mL}$ 二氯甲烷 萃取三次, 合并有机相依次用蒸馏水洗, 饱和食盐水洗, 无水硫酸镁干燥, 过滤除去干燥剂, 蒸除有机溶剂, 并 按下面两个步骤操作: (1)以 $V$ (乙酸乙酯) $: ~ V$ (石油醚) $=$ $1: 10$ 对 4-差基苯甲醛异戊烯基化后的混合物进行分离, 去除未反应的原料; (2)将不含原料的混合物在低于 $0{ }^{\circ} \mathrm{C}$ 环境中冷冻 $24 \mathrm{~h}$, 析出白色固体 $667.0 \mathrm{mg}(0.26 \mathrm{mmol})$, 产率 10\%. m.p. 99 101 ${ }^{\circ} \mathrm{C}$ (石油梄); ${ }^{1} \mathrm{H} \mathrm{NMR}\left(\mathrm{CDCl}_{3}\right.$, $400 \mathrm{MHz}) \delta$ : 9.81 (s, 1H, CHO), 7.53 (s, 2H, H-2 and 6), $6.13(\mathrm{~s}, 1 \mathrm{H}, \mathrm{OH}), 5.29 \sim 5.34\left(\mathrm{~m}, 2 \mathrm{H}, \mathrm{H}-2^{\prime}\right.$ and $\left.2 "\right), 3.21(\mathrm{~d}$, $J=7.2 \mathrm{~Hz}, 4 \mathrm{H}, \mathrm{H}-1^{\prime}$ and $1 "$ ), 1.79, 1.77 (each s, each $6 \mathrm{H}$, H-4', 4", 5' and 5"); IR (KBr) $v_{\text {max }}$ : 3200, 2967, 2920, 1655, 1580, 1443, 1280, $1125 \mathrm{~cm}^{-1}$; EI-MS (70 eV) $\mathrm{m} / z$ (\%): 258 
<smiles>COc1ccc(C(C)=O)c(O)c1</smiles><smiles>COc1cc(O)c(C(C)=O)c(OC)c1</smiles>

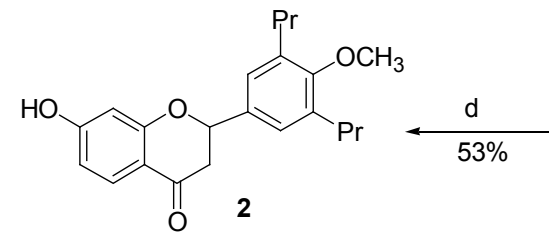<smiles>CCCc1cc(/C=C/C(=O)c2ccc(O)cc2O)cc(CCC)c1OC</smiles><smiles>CC(C)c1cc(O)ccc1C=O</smiles><smiles>CCCCPc1cc(C=O)cc(CCC)c1O</smiles><smiles>CCCc1cc(C=O)cc(P)c1OC</smiles><smiles>CCCc1cc(/C=C/C(=O)c2ccc(OC)cc2O)cc(CCC)c1OC</smiles>
5<smiles>CCCc1cc(C=O)cc2c1OC(C)(C)C=C2</smiles><smiles>CCC</smiles>

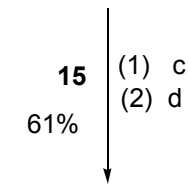

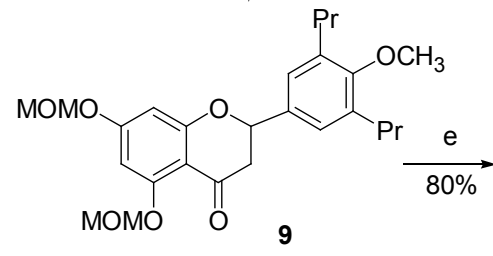<smiles>CCCc1cc(C2CC(=O)c3c(O)cc(O)cc3O2)cc(CCC)c1OC</smiles><smiles>CCCc1cc(C2CC(=O)c3c(OC)cc(OC)cc3O2)cc2c1OC(C)(C)C=C2</smiles>

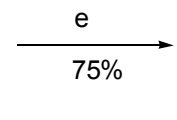<smiles>CCCc1cc(C2CC(=O)c3c(O)cc(O)cc3O2)cc2c1OC(C)(C)C=C2</smiles>

Reagents and conditions: (a) isoprenyl bromide, $\mathrm{KOH}, \mathrm{H}_{2} \mathrm{O}$; (b) dimethyl sulfate, anhydrous $\mathrm{K}_{2} \mathrm{CO}_{3}$, acetone, reflux; (c) $\mathrm{KOH}-\mathrm{H}_{2} \mathrm{O}-\mathrm{EtOH}, \mathrm{N}_{2}$; (d) $\mathrm{EtOH}, \mathrm{NaOAc}$, reflux; (e) $\mathrm{MeOH}, \mathrm{HCl}$, reflux; (f) $\mathrm{DDQ}$, benzene, reflux; (g) anhydrous $\mathrm{K}_{2} \mathrm{CO}_{3}$, acetone, $\mathrm{MOMCl}$, reflux

\section{Scheme 1}

$\left(\mathrm{M}^{+}, 63\right), 243$ (7), 229 (20), 187 (100); HRMS calcd for $\mathrm{C}_{17} \mathrm{H}_{22} \mathrm{O}_{2} 258.1620$, found 258.1618 .

\subsubsection{4-甲氧基-3, 5-二异戊烯基苯甲醛(7)的合成}

将化合物 $6258.0 \mathrm{mg}(1.0 \mathrm{mmol})$ 溶于 $10.0 \mathrm{~mL}$ 干燥 丙酩中，搅拌下加入无水碳酸钾 $304.0 \mathrm{mg}$ (2.2 mmol), 加热回流 $10 \mathrm{~min}$ 后, 缓慢滴加硫酸二甲酯 $0.2 \mathrm{~mL}(2.2$ $\mathrm{mmol}$ ), 继续搅拌 $30 \mathrm{~min}$ 后, 减压蒸除溶剂, 剩余物加 水溶解, 乙酸乙酯萃取 $(20 \mathrm{~mL} \times 3)$, 合并有机层并依次 用水洗, 饱和食盐水洗, 无水硫酸镁干燥, 过滤除去干 燥剂, 减压蒸除溶剂, 用 $V($ 乙酸乙酯 $): V($ 石油醚 $)=1$ :
10 为洗脱剂, 硅胶柱层析得无色油状物 $7267.0 \mathrm{mg}(0.98$ $\mathrm{mmol})$, 产率 98\%. ${ }^{1} \mathrm{H} \mathrm{NMR}\left(\mathrm{CDCl}_{3}, 400 \mathrm{MHz}\right) \delta$ : 9.89 (s, $1 \mathrm{H}, \mathrm{CHO}), 7.56$ (s, 2H, H-2 and 6), 5.27 5.31 (m, 2H, H-2' and 2"), 3.79 (s, 3H, $\left.\mathrm{OCH}_{3}\right), 3.41(\mathrm{~d}, J=7.2 \mathrm{~Hz}, 4 \mathrm{H}$, H-1' and 1"), 1.75 (s, 12H, H-4', 4", 5' and 5"); IR (KBr) $v_{\max }: 2970,2920,1693,1590,1470,1380,1270,1120$ $\mathrm{cm}^{-1}$; EI-MS (70 eV) m/z (\%): $272\left(\mathrm{M}^{+}, 2\right), 257$ (10), 241 (23), 43 (100). HRMS calcd for $\mathrm{C}_{18} \mathrm{H}_{24} \mathrm{O}_{2} 272.1776$, found 272.1774 . 


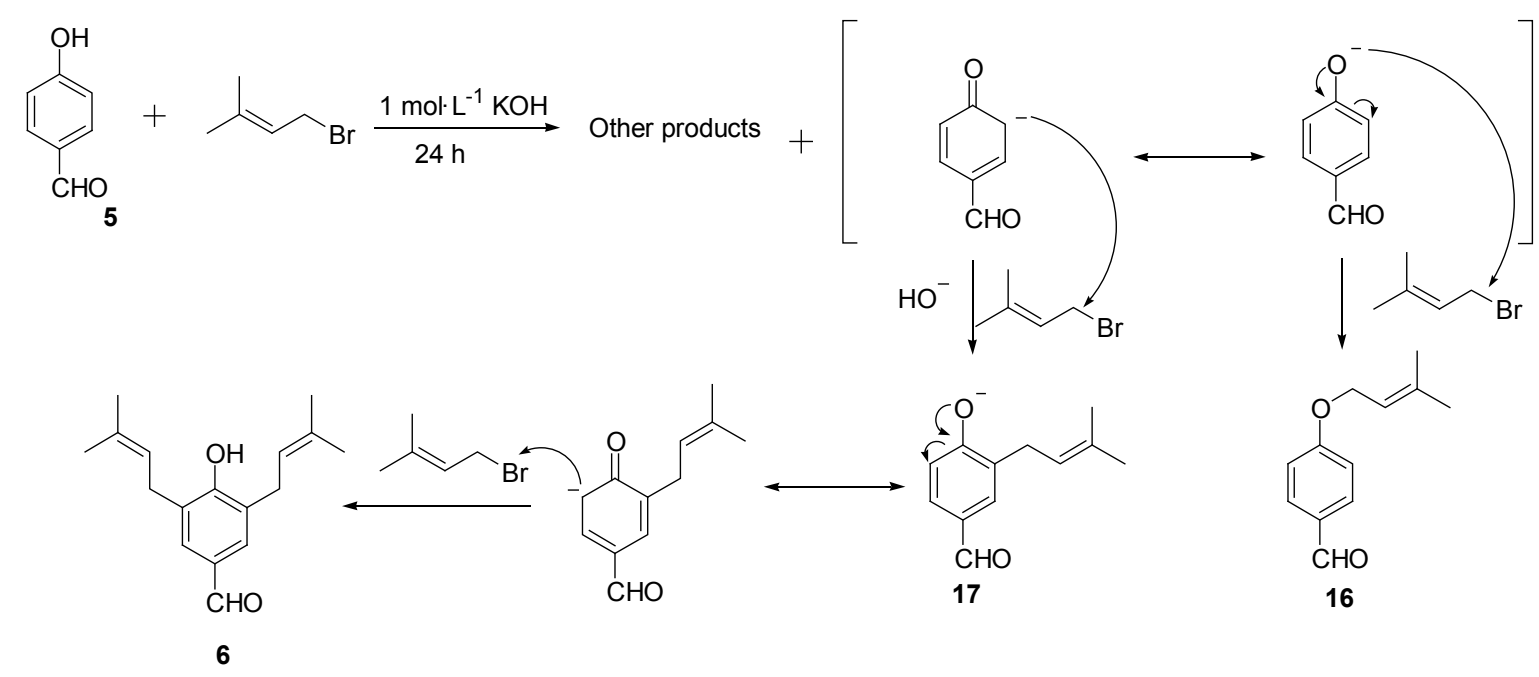

Scheme 2

2.2.3 2'-差基-4'-甲氧甲氧基-4-甲氧基-3，5-二异戊 烯基查尔酮 $(8)$ 的合成

将化合物 $7326.4 \mathrm{mg}$ (1.2 mmol)和化合物 13196.0 $\mathrm{mg}(1.0 \mathrm{mmol})$ 溶解到 $2.0 \mathrm{~mL}$ 乙醇中, 冷却至 $0{ }^{\circ} \mathrm{C}$, 缓慢 滴加 $\mathrm{KOH} 2.8 \mathrm{~g}(50.0 \mathrm{mmol})$ 的醇-水溶液 $6.7 \mathrm{~mL}$ $(V: V=3: 2)$, 氮气保护下 $0{ }^{\circ} \mathrm{C}$ 反应 $1 \mathrm{~h}$ 后, 自然恢复室 温继续反应 $24 \mathrm{~h}$, 将反应物倒入冰水中, 用 $3 \mathrm{~mol} \cdot \mathrm{L}^{-1}$ 的 盐酸调节 $\mathrm{pH}<2$, 用 $15.0 \mathrm{~mL}$ 二氯甲烷萃取三次, 合并 有机相并依次水洗, 饱和食盐水洗, 无水硫酸镁干燥, 过滤除去干燥剂, 减压蒸除溶剂, 剩余物用 $V($ 乙酸乙 酯)： $V$ (石油醚 $)=1: 8$ 为洗脱剂, 硅胶柱层析得黄色固 体 $8351.0 \mathrm{mg}(0.78 \mathrm{mmol})$, 产率 $78 \%$. ${ }^{1} \mathrm{H} \mathrm{NMR}\left(\mathrm{CDCl}_{3}\right.$, $400 \mathrm{MHz}) \delta: 13.35$ (s, 1H, OH), 7.85 (d, $J=8.8 \mathrm{~Hz}, 1 \mathrm{H}$, H-6'), 7.83 (d, $J=15.6 \mathrm{~Hz}, 1 \mathrm{H}, \mathrm{H}-\beta), 7.44$ (d, $J=15.6 \mathrm{~Hz}$, $1 \mathrm{H}, \mathrm{H}-\alpha), 7.33$ (s, 2H, H-2 and 6), 6.64 (d, $J=2.8 \mathrm{~Hz}, 1 \mathrm{H}$, H-3'), 6.60 (dd, $J=8.8,2.4 \mathrm{~Hz}, 1 \mathrm{H}, \mathrm{H}-5 '), 5.28 \sim 5.32(\mathrm{~m}$, $2 \mathrm{H}, \mathrm{H}-2^{\prime}$ and $\left.2^{\prime \prime}\right), 5.23\left(\mathrm{~s}, 2 \mathrm{H}, \mathrm{OCH}_{2} \mathrm{O}\right), 3.77$ (s, $3 \mathrm{H}$, $\left.\mathrm{OCH}_{3}\right), 3.49$ (s, 3H, $\left.\mathrm{OCH}_{3}\right), 3.40$ (d, $J=7.2 \mathrm{~Hz}, 4 \mathrm{H}, \mathrm{H}-1 "$ and $1 "$ "'); 1.77 (s, 12H, H-4", 4"', 5" and 5"'); IR (KBr) $v_{\max }$ : 2970, 2920, 1636, 1575, 1507, 1470, 1303, 1260, 1128; EI-MS (70 eV) m/z (\%): $450\left(\mathrm{M}^{+}, 22\right), 419$ (7), 405 (2), 45 (76), 43 (100); HRMS calcd for $\mathrm{C}_{28} \mathrm{H}_{34} \mathrm{O}_{5} 450.2406$, found 450.2408 .

$2.2 .45,7$-二甲氧甲氧基-4'- 甲氧基-3',5'-二异戊烯基 黄烷酮(9)的合成

将化合物 $7130.6 \mathrm{mg}$ (0.5 mmol)和化合物 15102.4 $\mathrm{mg}(0.4 \mathrm{mmol})$ 溶解到 $2.0 \mathrm{~mL}$ 乙醇中, 冷却至 $0{ }^{\circ} \mathrm{C}$, 缓慢 滴加 $\mathrm{KOH} 1.1 \mathrm{~g}(19.6 \mathrm{mmol})$ 的醇-水溶液 $5.0 \mathrm{~mL}$ $(V: V=3: 2)$, 氮气保护下 $0{ }^{\circ} \mathrm{C}$ 反应 $1 \mathrm{~h}$ 后, 自然恢复室 温继续反应 $24 \mathrm{~h}$, 将反应物倒入冰水中, 用 $3 \mathrm{~mol} \cdot \mathrm{L}^{-1}$ 的
盐酸调节 $\mathrm{pH}<2$, 用 $15.0 \mathrm{~mL}$ 二氯甲烷萃取三次，合并 有机相并依次水洗, 饱和食盐水洗, 无水硫酸镁干燥, 过滤除去干燥剂, 减压蒸除溶剂, 剩余物溶解到 $7.0 \mathrm{~mL}$ 无水乙醇中, 加入一滴水和无水醋酸钠 $656.0 \mathrm{mg}(8.0$ $\mathrm{mmol}$, 加热回流 $24 \mathrm{~h}$ 后, 冷却至室温, 加入少量水, 用 $20.0 \mathrm{~mL}$ 乙酸乙酯萃取 3 次, 合并有机层并依次用水洗, 饱和食盐水洗, 无水硫酸镁干燥, 过滤除去干燥剂, 减 压蒸除溶剂, 剩余物用 $V($ 乙酸乙酯 $): V($ 石油醚 $)=1$ : 10 为洗脱剂, 硅胶柱层析得淡黄色液体 $9124.0 \mathrm{mg}$ (0.24 mmol), 产率 $61 \%$. ${ }^{1} \mathrm{H} \mathrm{NMR}\left(\mathrm{CDCl}_{3}, 400 \mathrm{MHz}\right) \delta$ : $7.10\left(\mathrm{~s}, 2 \mathrm{H}, \mathrm{H}-2^{\prime}\right.$ and 6'), $6.43(\mathrm{~d}, J=2.0 \mathrm{~Hz}, 1 \mathrm{H}, \mathrm{ArH})$, $6.40(\mathrm{~d}, J=2.0 \mathrm{~Hz}, 1 \mathrm{H}, \mathrm{ArH}), 5.32(\mathrm{dd}, J=2.4,13.6 \mathrm{~Hz}$, $1 \mathrm{H}, \mathrm{H}-2), 5.27 \sim 5.29(\mathrm{~m}, 2 \mathrm{H}, \mathrm{H}-2 "$ and $2 " '), 5.27(\mathrm{~s}, 2 \mathrm{H}$, $\mathrm{OCH}_{2} \mathrm{O}$ ), 5.17 (s, 2H, $\left.\mathrm{OCH}_{2} \mathrm{O}\right), 3.74(\mathrm{~s}, 3 \mathrm{H}, \mathrm{OH}), 3.53$ (s, $\left.3 \mathrm{H}, \mathrm{OCH}_{3}\right), 3.47\left(\mathrm{~s}, 3 \mathrm{H}, \mathrm{OCH}_{3}\right), 3.39$ (d, J=7.2 Hz, 4H, H-1" and 1"'), 3.03 (dd, $J=13.6,16.4 \mathrm{~Hz}, 1 \mathrm{H}, \mathrm{H}-3_{\mathrm{ax}}$ ), 2.74 (dd, $J=2.8,16.4$ Hz, 1H, H-3 eq), 1.74 (s, 12H, H-4", 4"', 5" and 5"'); IR (KBr) v $v_{\max }$ : 2970, 2920, 1640, 1580, 1475, 1450, 1370, 1340, 1270, 1190, $1020 \mathrm{~cm}^{-1}$; EI-MS (70 eV) m/z (\%): $510\left(\mathrm{M}^{+}, 4\right), 479$ (10), 465 (7), 45 (100); HRMS calcd for $\mathrm{C}_{30} \mathrm{H}_{38} \mathrm{O}_{7} 510.2618$, found 510.2622.

2.2.5 2,2-二甲基-6-甲酰基-8-异戊烯基-2H-1-苯并吡 喃(10)的合成

搅拌下, 将 $129.0 \mathrm{mg}(0.5 \mathrm{mmol})$ 化合物 6 溶于 $3 \mathrm{~mL}$ 苯中, 剧烈搅拌下加入 DDQ $113.5 \mathrm{mg}(0.5 \mathrm{mmol})$, 然后 加热回流 $60 \mathrm{~min}$, 冷却至室温, 加入少量水, 乙酸乙酯 萃取 3 次, 合并有机相依次用蒸馏水洗、饱和的食盐水 洗, 无水硫酸镁干燥, 过滤除去干燥剂, 减压蒸去溶剂, 残余物用 $V$ (乙酸乙酯 $): V($ 石油醚 $)=1: 20$ 为洗脱剂, 硅胶柱层析得无色油状物 $1093.0 \mathrm{mg}$ (0.36 mmol), 产率 
73\%. ${ }^{1} \mathrm{H}$ NMR $\left(\mathrm{CDCl}_{3}, 400 \mathrm{MHz}\right) \delta: 9.79$ (s, $\left.1 \mathrm{H}, \mathrm{CHO}\right)$, $7.51(\mathrm{~d}, J=2.0 \mathrm{~Hz}, 1 \mathrm{H}, \mathrm{H}-6), 7.36(\mathrm{~d}, J=2.0 \mathrm{~Hz}, 1 \mathrm{H}$, H-2), 6.35 (d, $J=10 \mathrm{~Hz}, 1 \mathrm{H}, \mathrm{H}-4$ '), 5.67 (d, $J=10 \mathrm{~Hz}, 1 \mathrm{H}$, H-3'), $5.24 \sim 5.29$ (m, 1H, H-2"), 3.29 (d, $J=7.2 \mathrm{~Hz}, 2 \mathrm{H}$, H-1"), 1.73 (s, 6H, H-4" and 5"), 1.45 (s, 6H, H-5' and 6'); IR (KBr) $v_{\max }: 2970,2815,2720,1685,1590,1450,1375$, 1280, 1209, $1128 \mathrm{~cm}^{-1}$; EI-MS (70 eV) $\mathrm{m} / \mathrm{z}(\%): 256\left(\mathrm{M}^{+}\right.$, 15), 241 (100); HRMS calcd for $\mathrm{C}_{17} \mathrm{H}_{20} \mathrm{O}_{2} 256.1463$, found 256.1467.

2.2.6 5,7-二甲氧甲氧基-4',5'-(2,2-二甲基吡喃)-3'-异 戊烯基黄烷酮(11)的合成

将化合物 $10153.6 \mathrm{mg}(0.6 \mathrm{mmol})$ 和化合物 $\mathbf{1 5} 128.0$ $\mathrm{mg}(0.5 \mathrm{mmol})$ 溶解到 $2.0 \mathrm{~mL}$ 乙醇中, 冷却至 $0{ }^{\circ} \mathrm{C}$, 缓慢 滴加 $\mathrm{KOH} 1.4 \mathrm{~g}(25.0 \mathrm{mmol})$ 的醇一水溶液 $7.0 \mathrm{~mL}$ $(V: V=3: 2)$, 氮气保护下 $0{ }^{\circ} \mathrm{C}$ 反应 $1 \mathrm{~h}$ 后, 自然恢复室 温继续反应 $24 \mathrm{~h}$, 将反应物倒入冰水中, 用 $3 \mathrm{~mol} \cdot \mathrm{L}^{-1}$ 的 盐酸调节 $\mathrm{pH}<2$, 用 $15.0 \mathrm{~mL}$ 二氯甲烷萃取三次, 合并 有机相并依次水洗、饱和食盐水洗、无水硫酸镁干燥、 过滤除去干燥剂、减压蒸除溶剂, 剩余物溶解到 $5.0 \mathrm{~mL}$ 无水乙醇中, 加入一滴水和无水醋酸钠 $820.0 \mathrm{mg}(10.0$ $\mathrm{mmol}$ ), 加热回流 $24 \mathrm{~h}$ 后, 冷却至室温, 加入少量水, 用 $20.0 \mathrm{~mL}$ 乙酸乙酯萃取 3 次, 合并有机层并依次用水洗、 饱和食盐水洗、无水硫酸镁干燥、过滤除去干燥剂、减 压蒸除溶剂, 剩余物用 $V$ (乙酸乙酯) $: V$ (石油醚 $)=1$ :

10 为洗脱剂, 硅胶柱层析得淡黄色液体 $11121.0 \mathrm{mg}$ (0.24 mmol), 产率 49\%. ${ }^{1} \mathrm{H}$ NMR $\left(\mathrm{CDCl}_{3}, 400 \mathrm{MHz}\right) \delta$ : $7.02\left(\mathrm{~d}, J=2.0 \mathrm{~Hz}, 1 \mathrm{H}, \mathrm{H}-6^{\prime}\right), 6.93(\mathrm{~d}, J=2.0 \mathrm{~Hz}, 1 \mathrm{H}$, H-2'), 6.42 (d, $J=2.0 \mathrm{~Hz}, 1 \mathrm{H}, \mathrm{ArH}), 6.39$ (d, $J=2.4 \mathrm{~Hz}$, 1H, ArH), 6.32 (d, $J=9.6 \mathrm{~Hz}, 1 \mathrm{H}, \mathrm{H}-4 "), 5.63$ (d, $J=9.6$ $\mathrm{Hz}, 1 \mathrm{H}, \mathrm{H}-3 "), 5.29$ (dd, $J=2.4,13.2 \mathrm{~Hz}, 1 \mathrm{H}, \mathrm{H}-2), 5.28$ (s, $\left.2 \mathrm{H}, \mathrm{OCH}_{2} \mathrm{O}\right), 5.25 \sim 5.29\left(\mathrm{~m}, 1 \mathrm{H}, \mathrm{H}-2{ }^{\prime \prime}\right), 5.16(\mathrm{~s}, 2 \mathrm{H}$, $\left.\mathrm{OCH}_{2} \mathrm{O}\right), 3.53$ (s, $\left.3 \mathrm{H}, \mathrm{OCH}_{3}\right), 3.47$ (s, $\left.3 \mathrm{H}, \mathrm{OCH}_{3}\right), 3.28$ (d, $\left.J=7.2 \mathrm{~Hz}, 2 \mathrm{H}, \mathrm{H}-1{ }^{\prime \prime}\right), 3.03$ (dd, $J=13.2,16.4 \mathrm{~Hz}, 1 \mathrm{H}$, H-3 $3_{\text {ax }}$ ), 2.73 (dd, $J=2.4,16.4 \mathrm{~Hz}, 1 \mathrm{H}, \mathrm{H}-3_{\text {eq }}$ ), 1.73 (s, 6H, H-4" and 5"), 1.42 (s, 6H, H-5" and 6"); IR (KBr) $v_{\max }$ : 2970, 2920, 1630, 1606, 1582, 1357, 1257, 1156, 1074 $\mathrm{cm}^{-1}$; EI-MS (70 eV) m/z (\%): $494\left(\mathrm{M}^{+}, 1\right), 479$ (7), 449 (1), 239 (32), 45 (100); HRMS calcd for $\mathrm{C}_{29} \mathrm{H}_{34} \mathrm{O}_{7}$ 494.2305, found 494.2290.

\subsubsection{2-羟基-4-甲氧甲氧基苯乙酮(13)的合成}

将 2,4-二羟基苯乙酮 $304.0 \mathrm{mg}(2.0 \mathrm{mmol})$ 溶于 20.0 $\mathrm{mL}$ 干燥的丙酮中, 剧烈搅拌下加入无水碳酸钾 690.0 $\mathrm{mg}(5.0 \mathrm{mmol})$, 加热回流 $10 \mathrm{~min}$ 后, 缓慢滴加氯甲基 甲基醚 $320.0 \mathrm{mg}(4.0 \mathrm{mmol})$, 继续摚拌回流 $2 \mathrm{~h}$, 冷却至 室温, 减压蒸去溶剂, 残余物直接用 $V$ (乙酸乙酯):
$V($ 石油醚 $)=1: 10$ 为洗脱剂, 硅胶柱层析得淡黄色油状 物 $13365.0 \mathrm{mg}(1.86 \mathrm{mmol})$, 产率 $93 \%$. ${ }^{1} \mathrm{H} \mathrm{NMR}\left(\mathrm{CDCl}_{3}\right.$, $400 \mathrm{MHz}) \delta$ : 12.59 (s, 1H, OH), $7.63(\mathrm{~d}, J=9.2 \mathrm{~Hz}, 1 \mathrm{H}$, $\operatorname{ArH}), 6.57$ (d, $J=2.4 \mathrm{~Hz}, 1 \mathrm{H}, \operatorname{ArH}), 6.53$ (dd, $J=8.8,2.4$ $\mathrm{Hz}, 1 \mathrm{H}, \mathrm{ArH}), 5.19\left(\mathrm{~s}, 2 \mathrm{H}, \mathrm{OCH}_{2} \mathrm{O}\right), 3.46\left(\mathrm{~s}, 3 \mathrm{H}, \mathrm{OCH}_{3}\right)$, $2.55\left(\mathrm{~s}, 3 \mathrm{H}, \mathrm{COCH}_{3}\right)$; IR $(\mathrm{KBr}) v_{\text {max }}: 2963,1638,1505$, 1368, 1255, 1146, $995 \mathrm{~cm}^{-1}$; EI-MS (70 eV) $\mathrm{m} / z(\%): 196$ $\left(\mathrm{M}^{+}, 16\right), 181$ (1), 151 (7), 45 (100); HRMS calcd for $\mathrm{C}_{10} \mathrm{H}_{12} \mathrm{O}_{4}$ 196.0736, found 196.0739.

\subsubsection{2-着基-4, 6-二(甲氧甲氧基)苯乙酮(15)的合成}

将 2,4,6-三羟基苯乙酮 $336.0 \mathrm{mg}(2.0 \mathrm{mmol})$ 溶于 $20.0 \mathrm{~mL}$ 干燥的丙酮中, 剧烈搅拌下加入无水碳酸钾 $1.4 \mathrm{~g}(10.1 \mathrm{mmol})$, 加热回流 $10 \mathrm{~min}$ 后, 缓慢滴加氯甲基 甲基醚 $640.0 \mathrm{mg}(8.0 \mathrm{mmol})$, 继续摚拌回流 $2 \mathrm{~h}$, 冷却至 室温, 减压蒸去溶剂, 残余物直接用 $V$ (乙酸乙酯) : $V$ (石油醚 $)=1: 10$ 为洗脱剂, 硅胶柱层析得淡黄色油状 物 $15460.8 \mathrm{mg}(1.8 \mathrm{mmol})$, 产率 $90 \% .{ }^{1} \mathrm{H} \mathrm{NMR}\left(\mathrm{CDCl}_{3}\right.$, $400 \mathrm{MHz}) \delta: 13.71$ (s, 1H, OH), $6.23(\mathrm{~d}, J=2.4 \mathrm{~Hz}, 1 \mathrm{H}$, $\mathrm{ArH}), 6.21(\mathrm{~d}, J=2.4 \mathrm{~Hz}, 1 \mathrm{H}, \mathrm{ArH}), 5.23\left(\mathrm{~s}, 2 \mathrm{H}, \mathrm{OCH}_{2} \mathrm{O}\right)$, $5.14\left(\mathrm{~s}, 2 \mathrm{H}, \mathrm{OCH}_{2} \mathrm{O}\right), 3.49$ (s, $\left.3 \mathrm{H}, \mathrm{OCH}_{3}\right), 3.44(\mathrm{~s}, 3 \mathrm{H}$, $\mathrm{OCH}_{3}$ ), 2.63 (s, 3H, $\mathrm{COCH}_{3}$ ); IR (KBr) $v_{\max }: 2960,2925$, $1625,1435,1360,1265,1220,1150,1060 \mathrm{~cm}^{-1}$; EI-MS (70 eV) $\mathrm{m} / \mathrm{z}(\%): 256\left(\mathrm{M}^{+}, 9\right), 211$ (1), 182 (12), 45 (100); HRMS calcd for $\mathrm{C}_{12} \mathrm{H}_{16} \mathrm{O}_{6} 256.0947$, found 256.0948 .

2.2.9 (土)-Abyssinone-VI-4-O-methyl ether (1) 的合 成

将化合物 $890.0 \mathrm{mg}(0.2 \mathrm{mmol})$ 溶解到 $2.0 \mathrm{~mL}$ 甲醇 中, 摚拌下加入 $3 \mathrm{~mol} \cdot \mathrm{L}^{-1}$ 盐酸 $0.7 \mathrm{~mL}$, 加热回流 30 $\min$, 向溶液中加入 $1.0 \mathrm{~mL}$ 水, 用 $20.0 \mathrm{~mL}$ 乙酸乙酯萃 取三次, 合并有机层并依次水洗、饱和食盐水洗、无水 硫酸镁干燥、过滤除去干燥剂、减压蒸除溶剂, 剩余物 用 $V$ (乙酸乙酯) $: V($ 石油醚 $)=1: 10$ 为洗脱剂, 硅胶柱 层析得黄色固体 $167.4 \mathrm{mg}(0.17 \mathrm{mmol})$, 产率 83\%. m.p. $90 \sim 93{ }^{\circ} \mathrm{C}(\mathrm{MeOH}) ;{ }^{1} \mathrm{H}$ NMR $\left(\mathrm{CDCl}_{3}, 400 \mathrm{MHz}\right) \delta$ : 13.47 (s, 1H, OH), 7.84 (d, $\left.J=8.8 \mathrm{~Hz}, 1 \mathrm{H}, \mathrm{H}-6^{\prime}\right), 7.82$ (d, $J=16 \mathrm{~Hz}, 1 \mathrm{H}, \mathrm{H}-\beta), 7.43(\mathrm{~d}, J=15.6 \mathrm{~Hz}, 1 \mathrm{H}, \mathrm{H}-\alpha), 7.32$ (s, 2H, H-2 and 6), 6.45 (dd, $J=2.4,8.8 \mathrm{~Hz}, 1 \mathrm{H}, \mathrm{H}-5$ '), $6.43\left(\mathrm{~d}, J=2.4 \mathrm{~Hz}, 1 \mathrm{H}, \mathrm{H}-3{ }^{\prime}\right), 5.28 \sim 5.32\left(\mathrm{~m}, 2 \mathrm{H}, \mathrm{H}-2^{\prime \prime}\right.$ and 2"'), 3.77 (s, $3 \mathrm{H}, \mathrm{OCH}_{3}$ ), 3.39 (d, $J=7.2 \mathrm{~Hz}, 4 \mathrm{H}, \mathrm{H}-1 "$ and 1"'), 1.77 (s, 12H, H-4", 4"', 5" and 5"'); ${ }^{13} \mathrm{C}$ NMR $\left(\mathrm{CDCl}_{3}, 100 \mathrm{MHz}\right) \delta$ : $192.2(\mathrm{C}=\mathrm{O}), 166.5\left(\mathrm{C}-2^{\prime}\right), 162.9$ (C-4'), 158.8 (C-4), 145.0 (C- $\beta$ ), 135.8 (C-3 and 5), 133.3 (C-3" and 3"'), 132.1 (C-6'), 130.8 (C-1), 128.6 (C-2 and 6), 122.6 (C-2" and 2"'), 119.0 (C- $\alpha$ ), 114.6 (C-1'), 107.9 (C-5'), 103.9 (C-3'), 61.2 (OMe), 28.5 (C-1" and 1"'), 25.9 
(C-4" and 4"'), 18.1 (C-5" and 5"'); IR (KBr) $v_{\max }$ : 3300 , 2968, 2920, 1633, 1570, 1365, 1265, $1130 \mathrm{~cm}^{-1}$; EI-MS (70 eV) $m / z$ (\%): 406 (M+32), 391 (4), 375 (7), 43 (100); HRMS calcd for $\mathrm{C}_{26} \mathrm{H}_{30} \mathrm{O}_{4} 406.2144$, found 406.2136 .

2.2.10 ( \pm )-Abyssinone-IV-4'-O-methyl ether (2)的合 成

将化合物 $140.6 \mathrm{mg}(0.1 \mathrm{mmol})$ 溶解到 $3.0 \mathrm{~mL}$ 乙醇 中, 加入一滴水和无水醋酸钠 $164.0 \mathrm{mg}(2.0 \mathrm{mmol})$, 加 热回流 $24 \mathrm{~h}$ 后, 冷却至室温, 加入少量水, 用 $20.0 \mathrm{~mL}$ 乙酸乙酯萃取 3 次, 合并有机层并依次用水洗、饱和食 盐水洗、无水硫酸镁干燥、过滤除去干燥剂、减压蒸除 溶剂, 剩余物用 $V($ 乙酸乙酯 $): V($ 石油醚 $)=1: 10$ 为洗 脱剂, 硅胶柱层析得白色固体 $221.5 \mathrm{mg}$ (0.053 mmol), 产率 53\%. m.p. 72 73 ${ }^{\circ} \mathrm{C}(\mathrm{MeOH}) ;{ }^{1} \mathrm{H}$ NMR $\left(\mathrm{CDCl}_{3}\right.$, $400 \mathrm{MHz}) \delta$ : $7.85(\mathrm{~d}, J=8.4 \mathrm{~Hz}, 1 \mathrm{H}, \mathrm{H}-5), 7.11(\mathrm{~s}, 2 \mathrm{H}$, H-2' and 6'), 6.53 (dd, $J=2.4,8.4 \mathrm{~Hz}, 1 \mathrm{H}, \mathrm{H}-6), 6.45$ (d, $J=2.4 \mathrm{~Hz}, 1 \mathrm{H}, \mathrm{H}-8), 5.37$ (dd, $J=2.8,13.6 \mathrm{~Hz}, 1 \mathrm{H}, \mathrm{H}-2$ ), $5.27 \sim 5.30$ (m, 2H, H-2" and 2"'), $5.20(\mathrm{~s}, 1 \mathrm{H}, \mathrm{OH}), 3.75$ (s, $\left.3 \mathrm{H}, \mathrm{OCH}_{3}\right), 3.39$ (d, $J=6.8 \mathrm{~Hz}, 4 \mathrm{H}, \mathrm{H}-1$ " and 1"'), 3.04 (dd, $J=13.2,16.8 \mathrm{~Hz}, 1 \mathrm{H}, \mathrm{H}-3_{\mathrm{ax}}$ ), 2.79 (dd, $J=2.8,16.8$ $\mathrm{Hz}, 1 \mathrm{H}, \mathrm{H}-3_{\mathrm{eq}}$ ), 1.75 (s, 6H, H-4" and 4"'), 1.73 (s, 6H, $\mathrm{H}-5$ " and $\left.5^{\prime \prime \prime)}\right) ;{ }^{13} \mathrm{C} \mathrm{NMR}\left(\mathrm{CDCl}_{3}, 100 \mathrm{MHz}\right) \delta$ : $191.2(\mathrm{C}-4)$, 163.9 (C-7), 163.2 (C-9), 156.7 (C-4'), 135.5 (C-3' and 5'), 134.3 (C-1'), 133.0 (C-3" and 3"'), 129.5 (C-5), 126.0 (C-2' and 6'), 122.8 (C-2" and 2"'), 115.1 (C-10), 110.7 (C-6), 103.6 (C-8), 80.1 (C-2), 61.1 (OMe), 44.3 (C-3), 28.6 (C-1" and 1"'), 25.9 (C-4" and 4"'), 18.1 (C-5" and 5"'); IR $(\mathrm{KBr}) v_{\max }: 3200,2970,2920,1650,1600,1460,1370$, 1270, $1125 \mathrm{~cm}^{-1}$; EI-MS (70 eV) m/z (\%): $406\left(\mathrm{M}^{+}, 59\right)$, 375 (16), 243 (56), 137 (100), 43 (50); HRMS calcd for $\mathrm{C}_{26} \mathrm{H}_{30} \mathrm{O}_{4} 406.2144$, found 406.2140 .

2.2.11 ( \pm )-Abyssinone-V-4'-O-methyl ether (3)的合 成

将化合物 $974.4 \mathrm{mg}(0.15 \mathrm{mmol})$ 溶解到 $2.0 \mathrm{~mL}$ 甲醇 中, 搅拌下加入 $3 \mathrm{~mol} \cdot \mathrm{L}^{-1}$ 盐酸 $1.0 \mathrm{~mL}$, 加热回流 50 $\min$, 向溶液中加入 $1.0 \mathrm{~mL}$ 水, 用 $20.0 \mathrm{~mL}$ 乙酸乙酯萃 取三次, 合并有机层并依次水洗, 饱和食盐水洗, 无水 硫酸镁干燥, 过滤除去干燥剂, 减压蒸除溶剂, 剩余物 用 $V$ (乙酸乙酯) $: V$ (石油醚 $)=1: 8$ 为洗脱剂, 硅胶柱层 析得白色固体 $350.6 \mathrm{mg}(0.12 \mathrm{mmol})$, 产率 80\%. m.p. $146 \sim 147{ }^{\circ} \mathrm{C}(\mathrm{MeOH}) ;{ }^{1} \mathrm{H}$ NMR $\left(\mathrm{CDCl}_{3}, 400 \mathrm{MHz}\right) \delta$ : $12.06(\mathrm{~s}, 1 \mathrm{H}, \mathrm{OH}), 7.09$ (s, 2H, H-2' and 6'), 5.99 (d, $J=$ $2.0 \mathrm{~Hz}, 1 \mathrm{H}, \mathrm{ArH}), 5.98(\mathrm{~d}, J=2.0 \mathrm{~Hz}, 1 \mathrm{H}, \mathrm{ArH}), 5.33$ (dd, $J=2.8,13.2 \mathrm{~Hz}, 1 \mathrm{H}, \mathrm{H}-2), 5.26 \sim 5.30\left(\mathrm{~m}, 2 \mathrm{H}, \mathrm{H}-2^{\prime \prime}\right.$ and 2"'), 3.75 (s, $3 \mathrm{H}, \mathrm{OCH}_{3}$ ), 3.39 (d, $J=7.2 \mathrm{~Hz}, 4 \mathrm{H}, \mathrm{H}-1$ " and
1"'), 3.10 (dd, $\left.J=13.2,17.2 \mathrm{~Hz}, 1 \mathrm{H}, \mathrm{H}-3_{\mathrm{ax}}\right), 2.78$ (dd, $J=$ 2.8, $17.2 \mathrm{~Hz}, 1 \mathrm{H}, \mathrm{H}-3_{\mathrm{eq}}$ ), 1.75 (s, 6H, H-4" and 4"'), 1.73 (s, $6 \mathrm{H}, \mathrm{H}-5$ " and $5 " ') ;{ }^{13} \mathrm{C} \mathrm{NMR}\left(\mathrm{CDCl}_{3}, 100 \mathrm{MHz}\right) \delta: 196.2$ (C-4), 164.4 (C-7), 164.3 (C-5), 163.3 (C-9), 156.6 (C-4'), 135.4 (C-3' and 5'), 133.7 (C-1'), 133.0 (C-3" and 3"'), 125.8 (C-2' and 6'), 122.5 (C-2" and 2"'), 103.2 (C-10), 96.6 (C-6), 95.5 (C-8), 79.3 (C-2), 60.9 (OMe), 43.2 (C-3), 28.4 (C-1" and 1"'), 25.8 (C-4" and 4"'), 17.9 (C-5" and 5"'); IR (KBr) $v_{\max }$ : 3360, 2970, 2920, 1680, 1605, 1580, 1435, 1370, 1260, 1210, 1145, $1065 \mathrm{~cm}^{-1}$; EI-MS (70 eV) m/z (\%): $422\left(\mathrm{M}^{+}, 63\right), 391$ (86), 243 (59), 179 (47), 153 (100); HRMS calcd for $\mathrm{C}_{26} \mathrm{H}_{30} \mathrm{O}_{5}$ 422.2093, found 422.2095.

\subsubsection{2(士)-Sigmoidin E (4) 的合成}

将化合物 $1198.8 \mathrm{mg}(0.2 \mathrm{mmol})$ 溶解到 $2.0 \mathrm{~mL}$ 甲醇 中, 搅拌下加入 $3 \mathrm{~mol} \cdot \mathrm{L}^{-1}$ 盐酸 $1.4 \mathrm{~mL}$, 加热回流 50 $\mathrm{min}$, 向溶液中加入 $1.0 \mathrm{~mL}$ 水, 用 $20.0 \mathrm{~mL}$ 乙酸乙酯萃 取三次, 合并有机层并依次水洗, 饱和食盐水洗, 无水 硫酸镁干燥, 过滤除去干燥剂, 减压蒸除溶剂, 剩余物 用 $V$ (乙酸乙酯 $): V($ 石油醚 $)=1: 10$ 为洗脱剂, 硅胶柱 层析得白色固体 $460.9 \mathrm{mg}(0.15 \mathrm{mmol})$, 产率 $75 \%$. ${ }^{1} \mathrm{H}$ NMR $\left(\mathrm{CDCl}_{3}, 400 \mathrm{MHz}\right) \delta: 12.07(\mathrm{~s}, 1 \mathrm{H}, \mathrm{OH}), 7.02(\mathrm{~d}$, $\left.J=2.4 \mathrm{~Hz}, 1 \mathrm{H}, \mathrm{H}-6^{\prime}\right), 6.92$ (d, $\left.J=2.0 \mathrm{~Hz}, 1 \mathrm{H}, \mathrm{H}-2^{\prime}\right), 6.31$ (d, $J=9.6 \mathrm{~Hz}, 1 \mathrm{H}, \mathrm{H}-4 "), 5.99$ (d, $J=2.0 \mathrm{~Hz}, 1 \mathrm{H}, \mathrm{ArH})$, $5.98(\mathrm{~d}, J=2.0 \mathrm{~Hz}, 1 \mathrm{H}, \operatorname{ArH}), 5.64(\mathrm{~d}, J=9.6 \mathrm{~Hz}, 1 \mathrm{H}$, H-3"), 5.29 (dd, $J=2.8,13.2 \mathrm{~Hz}, 1 \mathrm{H}, \mathrm{H}-2), 5.25 \sim 5.28$ (m, 1H, H-2"'), 3.28 (d, $\left.J=7.6 \mathrm{~Hz}, 2 \mathrm{H}, \mathrm{H}-1{ }^{\prime \prime}\right), 3.11$ (dd, $J=$ $\left.13.2,17.2 \mathrm{~Hz}, 1 \mathrm{H}, \mathrm{H}-3_{\mathrm{ax}}\right), 2.76$ (dd, $J=2.8,17.2 \mathrm{~Hz}, 1 \mathrm{H}$, $\mathrm{H}-3_{\mathrm{eq}}$ ), 1.73 (s, 6H, H-4" and 5"), 1.43 (s, 6H, H-5" and 6"); ${ }^{13} \mathrm{C} \mathrm{NMR}\left(\mathrm{CDCl}_{3}, 100 \mathrm{MHz}\right) \delta: 196.4$ (C-4), 164.5 (C-7), 164.3 (C-5), 163.4 (C-9), 151.1 (C-4'), 132.4 (C-3"'), 131.1 (C-3" and 2"'), 129.6 (C-1' and 3'), 127.5 (C-2'), 122.2 (C-4" and 6'), 121.1 (C-5'), 103.2 (C-10), 96.6 (C-6), 95.6 (C-8), 79.4 (C-2), 76.5 (C-2"), 43.1 (C-3), 28.2 (C-1"'), 28.1 (C-5" and 6"), 25.8 (C-4"'), 17.9 (C-5"'); IR (KBr) $v_{\max }: 3200,2970,2920,1635,1594,1500,1460,1340$, 1265, 1150, $1080 \mathrm{~cm}^{-1}$; EI-MS(70 eV) $m / z(\%): 406\left(\mathrm{M}^{+}\right.$, 18), 391 (100), 239 (18), 153 (9); HRMS calcd for $\mathrm{C}_{25} \mathrm{H}_{26} \mathrm{O}_{5}{ }^{+} 406.1780$, found 406.1781 .

\section{References}

[1] Salvatore, M. J.; Kng, A. B.; Graham A. C.; Onishi, H. R.; Bartizal, K. F.; Abruzzo, G. K.; Gill, C. J.; Ramjit, H. G.; Pitzenberger, S. M.; Witherup, K. M. J. Nat. Prod. 1998, 61, 640.

[2] Rahman, M. M.; Gray, A. I.; Khondkar, P.; Sarker, S. D. Pharm. Biol. 2008, 46, 356.

[3] Meragelman, T. L.; Tucker, K. D.; McClord, T. G.; Cardel-lina, J. 
H.; Shoemker, R. H. J. Nat. Prod. 2005, 68, 1790.

[4] Hirpara, K. V.; Aggarwal, P.; Mukherjee, A. J.; Joshi, N.; Burman, A. C. Curr. Med. Chem. 2009, 9, 138.

[5] Seshadri, T. R. Tetrahedron 1959, 6, 169.

[6] Yan, X.; Liu, H. Q.; Zou, Y. Q.; Ren, Z. H. Chin. J. Org. Chem. 2008, 28, 1534 (in Chinese).

(延胥, 刘会青, 邹永青, 任占华, 有机化学, 2008, 28, 1534.)

[7] Oliver-Bever, B. Medicinal Plants in Tropical West Africa, Cambridge University Press, New York, 1981, p. 100.

[8] Na, M.; Jang, J.; Njamen, D.; Mbafor, J. T.; Fomum, Z. T.; Kim, B. Y.; Oh, W. K.; Ahn, J. S. J. Nat. Prod. 2006, 69, 1572.

[9] Moriyasu, M.; Ichimaru, M.; Nishiyama, Y.; Kato, A.; Mathenge, S. G; Juma, F. D.; Nganga, J. N. J. Nat. Prod. 1998, 61, 185.

[10] Yenesew, A.; Midiwo, J. O.; Miessner, M.; Heydenreich, M.; Peter, M. G. Phytochemistry 1998, 48, 1439.

[11] Promsattha, R.; Tempesta, M. S.; Fomum, Z. T.; Mbafor, J. T. J.
Nat. Prod. 1988, 51, 611.

[12] Zhang, Y. H.; Yang, J. H.; Li, H. J.; Jiang, S. Z.; Li, Y. F.; Liu, W. Y. Chin. J. Chem. 2011, 29, 521.

[13] Yang, J. H.; Li, H. J.; Zhang, Y. H.; Jiang, S. Z.; Li, Y. F.; Xue, P.; Ma, Y. L.; Liu, W. Y. Chin. J. Org. Chem. 2011, 31, 1230 (in Chinese).

(杨金会, 李红俊, 张玉恒, 江世智, 李云峰, 薛屏, 马玉龙, 刘 万毅, 有机化学, 2011, 31, 1230.)

[14] Yang, J. H.; Luo, J. S.; Guo, D. D.; Huang, W. Q. Chin. J. Org. Chem. 2012, 32, 1749 (in Chinese). (杨金会, 落俊山, 郭冬冬, 黄文倩, 有机化学, 2012, 32, 1749.)

[15] Farmer, R. L.; Biddle, M. M.; Nibbs, A. E.; Huang, X. K.; Bergan, R. C.; Scheidt, K. A. ACS Med. Chem. Lett. 2010, 1, 400.

[16] Kazuaki, k.; Katsuo, H.; Sadakazu, Y.; Teruya, S.; Ichiro, T. S. Agric. Biol. Chem. 1975, 39, 133.

(Cheng, F.) 